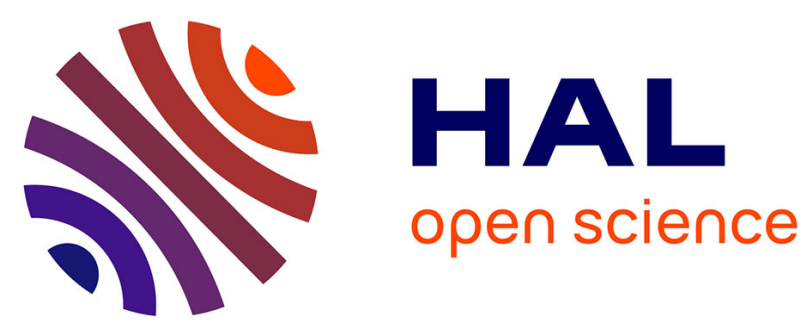

\title{
Update of an occupational asthma-specific job exposure matrix to assess exposure to 30 specific agents
}

Nicole Le Moual, Jan-Paul Zock, Orianne Dumas, Theodore Lytras, Eva Andersson, Linnéa Lillienberg, Vivi Schlünssen, Geza Benke, Hans Kromhout

\section{- To cite this version:}

Nicole Le Moual, Jan-Paul Zock, Orianne Dumas, Theodore Lytras, Eva Andersson, et al.. Update of an occupational asthma-specific job exposure matrix to assess exposure to 30 specific agents. Occupational and Environmental Medicine, 2018, Epub ahead of print. 10.1136/oemed-2017-104866 . inserm-01799430

\section{HAL Id: inserm-01799430 https://www.hal.inserm.fr/inserm-01799430}

Submitted on 24 May 2018

HAL is a multi-disciplinary open access archive for the deposit and dissemination of scientific research documents, whether they are published or not. The documents may come from teaching and research institutions in France or abroad, or from public or private research centers.
L'archive ouverte pluridisciplinaire HAL, est destinée au dépôt et à la diffusion de documents scientifiques de niveau recherche, publiés ou non, émanant des établissements d'enseignement et de recherche français ou étrangers, des laboratoires publics ou privés. 
Update of an Occupational Asthma-specific Job-Exposure Matrix to assess exposure to 30 specific agents

Nicole Le Moual ${ }^{1,2}$, Jan-Paul Zock ${ }^{3,4,5}$, Orianne Dumas ${ }^{1,2}$, Theodore Lytras ${ }^{3,4}$, Eva Andersson ${ }^{6}$, Linnéa Lillienberg $^{6}$, Vivi Schlünssen ${ }^{7}$, Geza Benke ${ }^{8}$, Hans Kromhout ${ }^{9}$

${ }^{1}$ Inserm, U1168, VIMA: Aging and chronic diseases. Epidemiological and public health approaches, F-94807, Villejuif, France

${ }^{2}$ Univ Versailles St-Quentin-en-Yvelines, UMR-S 1168, F-78180, Montigny Le Bretonneux, France

${ }^{3}$ Barcelona Institute of Global Health (ISGlobal), Barcelona, Spain

${ }^{4}$ Universitat Pompeu Fabra (UPF), Barcelona, Spain

${ }^{5}$ CIBER Epidemiología y Salud Pública (CIBERESP), Madrid, Spain

${ }^{6}$ Department of Occupational and Environmental Medicine, Sahlgrenska University Hospital, Gothenburg, Sweden

${ }^{7}$ Department of Public Health, Danish Ramazzini Centre, Aarhus University and National Research Center for the Working Environment, Copenhagen, Denmark

${ }^{8}$ Monash Centre for Occupation and Environmental Health, School of Public Health and Preventive Medicine, Monash University, Melbourne, Australia

${ }^{9}$ Department of Environmental Epidemiology, Institute for Risk Assessment Sciences, Utrecht University, Utrecht, the Netherlands

\section{Correspondence}

Nicole Le Moual

Inserm U 1168 / VIMA

Aging and chronic diseases. Epidemiological and public health approaches.

16, avenue Paul Vaillant Couturier

94807 Villejuif Cedex, France

e-mail: nicole.lemoual@inserm.fr

Tel: 33145595070

Fax: 33145595169

5 keywords : Asthma, Occupational exposure assessment, Job-Exposure Matrix, Asthmagens

Word count: up to 3500 words - 3669 words

Structured abstract: up to 250 words (headings : Objectives, Methods, Results, and Conclusions) 249 words Tables/Illustrations: up to 5 - 4 tables

References: up to 40 (more may be allowable for systematic reviews) - 43 references 


\section{ABSTRACT}

Objectives: We aimed to update an asthmagen Job-Exposure Matrix (JEM) developed in the late 1990s. Main reasons were: the number of suspected and recognised asthmagens has since tripled; understanding of the etiologic role of irritants in asthma and methodological insights in application of JEMs have emerged in the period.

Methods: For each agent of the new Occupational Asthma-specific JEM (OAsJEM), a working group of three experts out of eight evaluated exposure for each ISCO- 88 job code into three categories: 'high' (high probability of exposure and moderate-to-high intensity), 'medium' (low-to-moderate probability or low intensity) and 'unexposed'. Within a working group, experts evaluated exposures independently from each other. If expert assessments were inconsistent the final decision was taken by consensus. Specificity was favoured over sensitivity, i.e. jobs were classified with high exposure only if the probability of exposure was high and the intensity moderate-to-high. In the final review, all experts checked assigned exposures and proposed/improved recommendations for expert re-evaluation after default application of the JEM.

Results: The OAsJEM covers exposures to 30 sensitisers//irritants, including 12 newly recognized, classified into 7 broad groups. Initial agreement between the three experts was mostly fair-to-moderate (Kappa's values: 0.2-0.5). Out of 506 ISCO-88 codes, the majority was classified as unexposed (from $82.6 \%$ (organic solvents) to $99.8 \%$ (persulphates)) and a minority as 'high-exposed' $(0.2 \%$ (persulphates) to $2.6 \%$ (organic solvents)).

Conclusions: The OAsJEM, developed to improve occupational exposure assessment, may improve evaluations of associations with asthma in epidemiological studies and contribute to assessment of the burden of work-related asthma. 


\section{WHAT THIS PAPER ADDS?}

- What is already known about this subject?

To evaluate the burden of work-related asthma in community-based epidemiological studies, assessment of occupational exposures is a crucial issue.

An asthma-specific job-exposure-matrix (JEM) was constructed in the late 1990s and applied successfully in various studies on asthma or other immunological diseases. However, the number of identified asthmagens has tripled since the end of 1990s and an update of this JEM is warranted.

- What are the new findings?

A new Occupational Asthma-specific JEM (OAsJEM) was created to evaluate exposure to 30 specific agents, based on consensus from international experts in the field.

- How might this impact on policy or clinical practice in the foreseeable future?

This new OAsJEM, developed to improve exposure assessment to occupational asthmagens, may be a valuable tool to improve evaluations of associations between occupational exposures and asthma phenotypes in epidemiological studies, and contribute to assessment of the burden of disease due to exposure to occupational asthmagens. 


\section{INTRODUCTION}

Work-related asthma is the most common occupational lung disease in industrialized countries ${ }^{1}$. The proportion of asthma in adults attributable to occupational exposures is estimated to be around $15 \%{ }^{2}$. The burden of work-related asthma is underestimated partly due to limited knowledge about occupational exposure to well-established asthmagens ${ }^{3}$. In addition, new asthmagens are constantly being reported ${ }^{4}$. Occupational asthma is classically described as induced by a sensitizer (immunological asthma) or by a unique high level accidental exposure to an irritant agent (nonimmunological asthma) at work ${ }^{1,3}$. However, recently also chronic low-to-moderate level of exposure to irritants has been recognized as a cause of work-related asthma ${ }^{5-9}$. Underlying biological mechanisms are complex and partly unknown especially for reactive chemicals and irritants ${ }^{3,10}$. It has been suggested that irritant-induced asthma may represent between $10 \%$ and $20 \%$ of occupational asthma, with an increase in recent years ${ }^{3,11}$.

The deleterious role of cleaning agents and disinfectants in asthma has been recognized in recent decades $^{2,12,13}$. Other potential risk factors for asthma have received more attention recently especially occupational exposures to endotoxins, organic solvents, pesticides and chronic exposure to irritants in general $^{1,5,14-17}$. Studying associations between such kinds of agents, which may be sensitizers (low molecular weight chemicals) and/or lung irritants, and asthma is important for a better understanding of the disease $e^{3,10,12,17}$. Improvement of exposure assessment, especially for newly-recognized agents, is a crucial but challenging issue ${ }^{3,18}$ when studying work-related asthma in community-based cohorts.

Kennedy et al. ${ }^{19}$ proposed at the end of the last century an asthma-specific JEM combined with an expert step, to evaluate occupational exposures to common asthmagens ${ }^{20}$. This method, by favouring specificity over sensitivity via an expert re-evaluation step, improved asthmagen assessment and reduced misclassification errors ${ }^{19}$. In some studies limited improvement of the additional expert reevaluation step was observed, possibly due to imprecise available information regarding tasks ${ }^{21}$. This asthma-specific JEM is the most commonly used method in various populations both in and outside Europe $^{19}$ and has been adapted to country-specific workplace environments in Northern Europe and the $\mathrm{UK}^{15,22-24}$. Since the Asthma-specific JEM was developed the number of suspected asthmagens has tripled $^{3,4}$.

We aimed to develop a new Occupational Asthma-specific JEM (OAsJEM), combined with an expert re-evaluation step, to assess exposure to 30 specific sensitizers and/or irritants. 


\section{METHODS}

\section{The original Asthma-specific JEM}

Briefly, the original Asthma-specific JEM ${ }^{19}$, is a two dimensional table with a 2- to 4-digit ISCO-88 (International Standard Classification of Occupations, 1988) job codes ${ }^{25}$ axis crossed with a second axis of 18 asthmagens and four agents classified at low risk for asthma. Agents were classified into four broad groups: low molecular weight (LMW), high molecular weight (HMW) asthmagens, mixed environments and irritants peaks. In addition, job codes poorly defined and/or with heterogeneous exposures (88 out of all 506 2- to 4-digit ISCO-88 job codes; 17\%) were identified and classified as requiring 'an expert re-evaluation step' with linked comments and specific recommendations for reviewing each specific exposure (see online supplement Figure E1). When re-evaluation was needed in a study, the exposure attributed by the JEM was checked and modified if necessary by the expert directly, at individual level, according to participant's job, industry and tasks descriptions.

Two major strengths of this method were: (i) specificity favoured over sensitivity, i.e. a job was classified as exposed to a specific asthmagen only if the probability to be exposed was high for an important number of subjects in that job. (ii) an expert re-evaluation step, performed after applying the JEM, for an a priori list of jobs (poorly defined or with heterogeneous exposures) ${ }^{19}$. The JEM combined with expert re-evaluation step provided a more specific exposure assessment, by correcting for heterogeneity of exposures within occupations and thereby avoiding a classical drawback of most JEMs, and by so doing reducing the impact of misclassification error.

The asthma-specific JEM is commonly used (see online supplement Table E1 for more detail) and is freely available on a website (http://asthmajem.vjf.inserm.fr/), which was developed and launched in Vancouver in 2005 and hosted in France since 2011.

\section{The new Occupational Asthma-specific JEM (OAsJEM)}

A standardised procedure was used to develop the updated OAsJEM. First the list of agents was updated from the previous one (22 agents, categorised from a list of 150 substances from Chan-Yeung and $\mathrm{Malo}^{20}$ ) based on the recent literature ${ }^{1,3,5,7,26-28}$. The list was further improved during the process (Online supplement, Table E2) resulting in a final version consisting of 7 large groups with 30 specific agents in total (Table 1). The new agents were identified based on recent literature knowledge, using mostly information from two major literature reviews ${ }^{1,5}$ for sensitiser and irritant -induced asthma, respectively.

A working group of three experts was assigned to each agent: each expert evaluated exposure to a number of specific agents varying from 3 to 19 agents, according to their agent of choice and expertise. 
Table 1 - List of individual agents classified in 7 large groups - New Occupational Asthma-specific JEM

\begin{tabular}{|c|c|c|c|c|c|c|c|}
\hline Agents, $n=30$ & $\begin{array}{c}\text { HMW } \\
\text { sensitizer* }\end{array}$ & Mites & $\begin{array}{l}\text { Microbial } \\
\text { exposure* }\end{array}$ & $\begin{array}{c}\text { LMW } \\
\text { sensitizer* }\end{array}$ & Irritants* & $\begin{array}{c}\text { Highly } \\
\text { reactive } \\
\text { chemicals }\end{array}$ & Biocides \\
\hline Animals\# & 1 & & & & & & \\
\hline Fish/ Shellfish & 1 & & & & & & \\
\hline Flour & 1 & & & & & & \\
\hline Foods\# & 1 & & & & & & \\
\hline Plants-related dusts\# & 1 & & & & & & \\
\hline House dust Mites & 1 & 1 & & & & & \\
\hline Storage Mites & 1 & 1 & & & & & \\
\hline Plant Mites & 1 & 1 & & & & & \\
\hline Enzymes & 1 & & & & & & \\
\hline Latex & 1 & & & & & & \\
\hline Textile \# & 1 & & 1 & & 1 & & \\
\hline Moulds & & & 1 & & 1 & & \\
\hline Endotoxin & & & 1 & & 1 & & \\
\hline Drugs\# & 1 & & & 1 & & & \\
\hline $\begin{array}{l}\text { High level chemical } \\
\text { disinfectants \# }\end{array}$ & & & & 1 & 1 & 1 & 1 \\
\hline Aliphatic Amines & & & & 1 & 1 & 1 & \\
\hline Isocyanates & & & & 1 & 1 & 1 & \\
\hline Acrylates & & & & 1 & 1 & 1 & \\
\hline Epoxy resins & & & & 1 & 1 & 1 & \\
\hline Persulphates/Henna & & & & 1 & 1 & 1 & \\
\hline Wood\# & 1 & & & 1 & 1 & & \\
\hline Metal & & & & 1 & 1 & & \\
\hline $\begin{array}{l}\text { Metal Working } \\
\text { Fluids }\end{array}$ & & & 1 & 1 & 1 & & \\
\hline Herbicides & & & & & 1 & & 1 \\
\hline Insecticides & & & & & 1 & & 1 \\
\hline Fungicides & & & & & 1 & & 1 \\
\hline Indoor cleaning\# & & & & & 1 & & \\
\hline Bleach & & & & & 1 & 1 & 1 \\
\hline Organic Solvents & & & & & 1 & 1 & \\
\hline Exhaust fumes & & & & & 1 & & \\
\hline
\end{tabular}

The 12 new agents (out of 30), identified in bold, were chosen based on the knowledge from recent literature ${ }^{1,5}$.

\# Animals: exposure to animals in agriculture industry, research labs - land mammals and birds ; Foods: work with milk, eggs, peanuts, tree nuts, soy, ... mostly powders (process used to obtain powder may affect allergenic properties) - Fish/Shellfish and Flour are not included in foods ; Other plants-related dusts : Flour is not included; Enzyme: those used as improver in bread dough, or in washing detergents manufacturing ...; Textile: work with cotton or synthetic fibers, which explain its classification in 3 groups (HMW, microbes, irritants); Drugs : mostly HMW agents ${ }^{19}$ (Kennedy, personal communication) but potentially also $\mathrm{LMW}^{1}$; Wood : mostly LMW agents ${ }^{19}$ but potentially also $\mathrm{HMW}^{1,27,28}$; High level chemical disinfectants: High level disinfectant or chemical sterilizing agents (eg, glutaraldehyde, chlorhexidine, ethylene oxide, hydrogen peroxide); Indoor cleaning: Cleaning products/ detergents or low/intermediate level disinfectants

7 large groups: High Molecular Weight sensitizers ( $\mathrm{n}=13)$, of which Mites is a sub-category (3 sensitizers); Microbial exposure $(\mathrm{n}=4)$; Low Molecular Weight sensitizers (LMW, $n=10)$; Chronic exposure to irritants $(\mathrm{n}=19$ agents including 9 agents also classified as LMW sensitizers because for these 9 agents both mechanisms are possible ${ }^{1,3,5,19}$ ), of which Highly reactive chemicals (8 agents) and Biocides (5 agents) are 2 sub-categories. 
Classification of exposures was refined from yes/no to semi-quantitative metrics i.e, high: high probability of exposure and moderate to high intensity; medium: low to moderate probability or low intensity of exposure, such as 'high probability and low intensity' or 'low probability and moderate to high intensity'; no: unlikely to be exposed (low probability and low intensity). Experts classified a job as 'high probability of exposure' when they considered that at least $50 \%$ of the workers were exposed. The expert re-evaluation recommendations were also improved with a checking for (i) ISCO code for selected jobs (mostly the larger 2- and 3-digit ISCO-88 codes) and (ii) exposure assessment for selected jobs (poorly defined and/or heterogeneous jobs). For the expert re-evaluation recommendations, period and country-specific work situations were taken into account, in addition to job, industry and tasks recorded by each participant.

In addition, we addressed important methodological points during the process: (a) all experts evaluated exposure independently from each other. In case of disagreement, the final decision was taken by consensus within the experts' team. (b) specificity was favoured over sensitivity for high level of exposure only (not for 'medium' exposure).

At the beginning, all ISCO job codes were classified as 0: 'non exposed', if these jobs were classified unlikely to be exposed according to three existing JEMs (Asthma-specific ${ }^{19}$, ALOHA ${ }^{29,30}$ and Northern ${ }^{15,26}$ ) or 1: 'exposure assessment warranted', if classified by one of these three JEMs as potentially exposed to asthmagens, irritants or dusts, gases and fumes. In a first step, for all job classified as 'exposure assessment warranted', for a given agent a first evaluation of exposure (high, medium, no) was attributed by each of the three experts independently. The experts decision were based on assessments from existing JEMs and especially the Asthma-specific, N-JEM and ALOHA $\mathrm{JEMs}^{15,19,29,30}$ and on recent literature. In a second step, all exposure assessments were reviewed and compared. If the assessment was unanimous and consistent with previous evaluations (for 'old' agents from the Asthma-specific JEM) the job was classified as such; if not (jobs with at least one expert disagreeing or inconsistent with the previous evaluation), we used a standardised procedure and send to the three experts : an EXCEL file including information on the 3 initial evaluations, an exposure rating proposed by NLM with comments/justifications, and opportunities for further comments/ justifications from each expert, who were asked to send back the file completed with comments on the proposal. If all experts agreed, a final rating was made; in case of disagreement, the final decision was taken by consensus after a discussion (using information from the file) with the three experts during a conference call or a face to face meeting. Decisions regarding evaluations and recommendations are illustrated for latex in the online supplement (Tables E3, E4). The final step consisted of a complete review and feedback by all experts on the assigned exposures, their consistency and expert-step recommendations on exposure and code checking.

Basic characteristics of the Asthma-specific JEM and the new OAsJEM are compared in Table 2. 
Table 2 - Comparison of basic characteristics of the Asthma-specific JEM and the new OAsJEM

\begin{tabular}{l|c|c|}
\hline & Asthma-specific JEM & New OAsJEM \\
\hline Number of agents or groups of agents & 22 & 30 \\
HMW sensitizer & 9 & 13 \\
LMW sensitizer & 5 & 10 \\
Mixed exposure & 3 & 4 \\
$\quad \begin{array}{l}\text { Microbial exposures } \\
\text { Irritants, high peaks }\end{array}$ & - & - \\
$\quad$ Irritants, chronic exposure & 3 & No/medium/high \\
Classification & No/yes & No \\
Exposure at low risk for asthma identified & Yes & Yes \\
Standardized expert re-evaluation step (revising & Yes & 3 per agent \\
job codes, agents) & & $(8$ in total) \\
Number of experts to assess each agent & and consultation of co-authors \\
\end{tabular}

\section{Data analysis}

The initial evaluations of exposure to a specific agent obtained by each of the three experts were independently compared. Comparisons of chance-corrected agreement between of three pairs of experts per agent were undertaken by estimating Cohen's Kappa. The agreement was interpreted as follow: poor $(<0)$, slight $(0-0.2)$, fair (0.2-0.4), moderate (0.4-0.6), substantial (0.6-0.8) and almost perfect (0.8-1.0) agreement ${ }^{31}$. Prevalence of jobs classified exposed and with an expert-re-evaluation step, for each agent, was assessed after the final assessment of exposure at ISCO- 88 code level. 


\section{RESULTS}

The revised occupational asthma-specific JEM (Table 1) included exposure evaluations to 30 specific asthmagens (HMW ( $\mathrm{n}=13)$ and LMW (n=10) agents) and exposure to irritants $(\mathrm{n}=19)$. The twelve new agents evaluated through the OAsJEM are marked in bold (such as acrylates, epoxy resins). Exposures were classified into seven broad groups (Table 1): HMW sensitizers, mites (sub-category of HMW), microbial exposures, LMW sensitizers, chronic exposure to irritants, highly reactive chemicals, biocides (last two sub-categories of irritants). Two agents were classified as both potentially HMW and LMW sensitizers (drugs, wood) as their classification was inconsistent in the literature ${ }^{1,19,27,28}$. Most LMW sensitizers were also classified as irritants. Compared to the previous Asthma-specific $\mathrm{JEM}^{19}$, 'mixed environments' and 'low risk exposure' as well as to 'high irritant peaks', 'environmental tobacco smoke' were no longer included in the OAsJEM (online supplement Table E2).

Values of initial agreement between two experts varied strongly by agent (Table 3; e.g. the 3 Kappa's values varied from 0.08 to 0.54 for fish/shellfish and from 0.21 to 0.29 for plants) and were mostly fair-to-moderate $(0.2-0.5)$. The Kappa's values varied overall from -0.01 to 1.00 . Agreement could not be estimated for agents added after the first step (three categories of mites and pesticides, online supplement Table E2). For mites, experts decided by consensus after comparison of their first evaluations to split them into three sub-categories. We also decided a posteriori to assess exposures to three sub-categories of pesticides (herbicides, insecticides, fungicides).

Table 4 illustrates the percentage of jobs classified as exposed at high or medium level and at high level for the 30 agents. Out of 5062 to 4 digit ISCO-88 job codes, agents classified as exposed ranged from $0.2 \%$ (persulphates/henna) to $17.4 \%$ (organic solvents) and as highly-exposed from $0 \%$ (acrylates, enzymes, storage mites) to $2.6 \%$ (organic solvents, exhaust fumes). For 14 agents, such as epoxy resins $(1.8 \%)$ and flour $(0.6 \%)$, less than $3 \%$ of the jobs were classified exposed at high or medium level. Overall, more jobs were classified exposed with the OAsJEM, compared to the original JEM (Table 4), mostly explained because specificity was not favoured to evaluate medium level. However, for high level exposure the percentage were mostly lower with the OAsJEM (0.2 to 2.6\%) than with the old JEM (0.2 to 5.5\%). Examples of jobs classified at high exposure level are given in the online supplement (Table E5). Overall, the percentage of jobs classified with an expert reevaluation by the new OAsJEM (Table 4) varied from $0 \%$ (persulphates/henna) to $8.1 \%$ (metal). Considering only the 390 4-digit ISCO-88 job codes (Table E6), after exclusion of the 116 less precise codes (2- and 3- digits), the percentages of jobs classified as highly exposed were similar. In addition, around $20 \%$ of the jobs (113 out of 506 codes, mostly 2- and 3-digit codes) were identified as 'code checking required'. 
Table 3 - Cohen's Kappa values - New Occupational Asthma-specific JEM (OAsJEM)

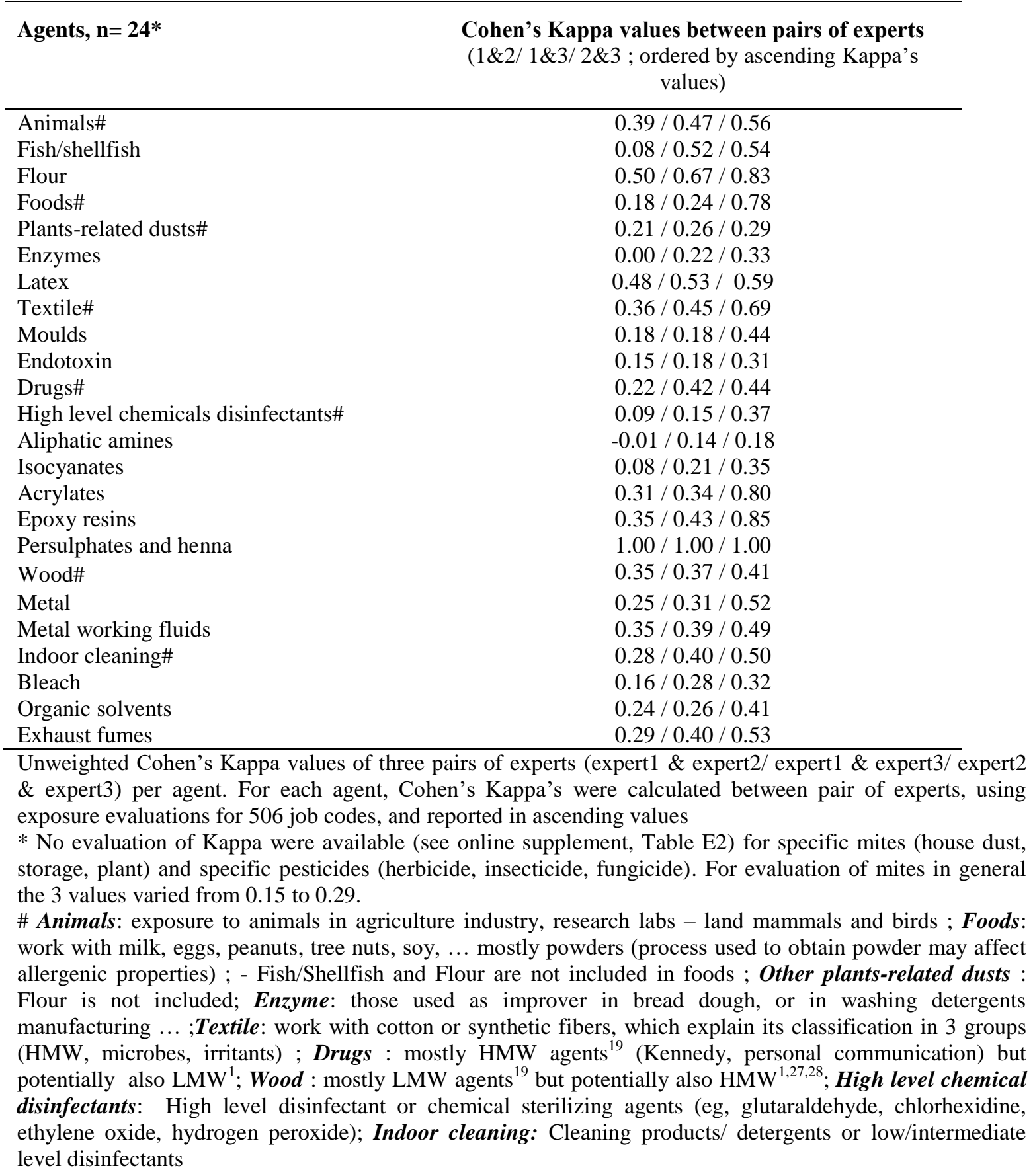


Table 4 - Percentage of jobs classified exposed or with an expert re-evaluation

\begin{tabular}{|c|c|c|c|c|}
\hline \multirow[b]{2}{*}{ Agents } & \multirow{2}{*}{$\begin{array}{c}\begin{array}{c}\text { Asthma-specific } \\
\text { JEM }\end{array} \\
\text { Exposed, \% }\end{array}$} & \multicolumn{3}{|c|}{ New OAsJEM } \\
\hline & & $\begin{array}{l}\text { Exposure } \\
\text { Medium or } \\
\text { High } \\
\end{array}$ & $\begin{array}{l}\% * \\
\text { High } \\
\text { only }\end{array}$ & $\begin{array}{c}\text { Expert re- } \\
\text { evaluation, } \% *\end{array}$ \\
\hline Animals & 2.2 & 3.4 & 1.2 & 4.2 \\
\hline Fish/shellfish & 0.2 & 2.2 & 0.8 & 1.8 \\
\hline Flour & 0.6 & 0.6 & 0.4 & 0.8 \\
\hline Foods & - & 1.0 & 0.2 & 1.8 \\
\hline Plants-related dusts & 0.8 & 3.4 & 0.6 & 4.9 \\
\hline House dust mites & $-* *$ & 0.8 & 0.2 & 1.2 \\
\hline Storage mites & $-* *$ & 3.4 & 0.0 & 0.8 \\
\hline Plant mites & $-* *$ & 2.8 & 0.4 & 4.3 \\
\hline Enzymes & 0.2 & 1.0 & 0.0 & 1.4 \\
\hline Latex & 2.2 & 4.2 & 1.2 & 4.5 \\
\hline Textile & 3.2 & 3.2 & 0.6 & 3.4 \\
\hline Moulds & 1.2 & 6.3 & 0.6 & 3.0 \\
\hline Endotoxin & - & 5.5 & 1.6 & 3.8 \\
\hline Drugs & 1.2 & 1.2 & 0.4 & 2.0 \\
\hline High level chemicals disinfectants & 0.8 & 7.1 & 0.6 & 4.7 \\
\hline Aliphatic Amines & - & 2.0 & 0.6 & 3.6 \\
\hline Isocyanates & 1.0 & 1.4 & 0.2 & 2.2 \\
\hline Acrylates & - & 2.0 & 0.0 & 3.2 \\
\hline Epoxy resins & - & 1.8 & 0.8 & 1.8 \\
\hline Persulphates/Henna & - & 0.2 & 0.2 & 0.0 \\
\hline Wood & 0.0 & 3.6 & 1.0 & 2.0 \\
\hline Metal & 5.5 & 9.3 & 1.4 & 8.1 \\
\hline Metal Working Fluids & 0.8 & 2.0 & 1.0 & 1.6 \\
\hline Herbicides & - & 3.2 & 1.4 & 2.0 \\
\hline Insecticides & - & 4.2 & 1.8 & 2.4 \\
\hline Fungicides & - & 4.0 & 1.8 & 1.0 \\
\hline Indoor cleaning & - & 4.2 & 0.6 & 1.8 \\
\hline Bleach & - & 2.2 & 0.4 & 1.6 \\
\hline Organic solvents & - & 17.4 & 2.6 & 5.5 \\
\hline Exhaust fumes & 4.0 & 11.9 & 2.6 & 7.1 \\
\hline
\end{tabular}

$*$ percentage of jobs classified exposed at high or medium level and at high level, respectively out of 506. For 14 agents, the percentages of jobs classified exposed were less than $3 \%$.

In the second column, in italic: percentage of jobs classified exposed by the original Asthma-specific JEM ${ }^{19}$. ** 0.4 for mites and insect antigen in general according to the original Asthma-specific JEM ${ }^{19}$ 


\section{DISCUSSION}

We developed an OAsJEM to update and to improve occupational exposure assessment for 30 specific asthmagens and irritants, classified into seven large groups, for 506 2- to 4-digit ISCO-88 job codes. The initial agreement observed between three experts per agent was fair-to-moderate in most cases and final decisions regarding exposure assessment were taken by consensus. These results stress the importance of evaluating occupational exposures among a working group of at least two experts. The expert re-evaluation step was improved and country and time specific issues (e.g. replacement of latex powered gloves) were taken into account.

\section{A priori or a posteriori choice of specific exposures evaluation}

Agents included in the category 'at low risk for asthma' in the original $\mathrm{JEM}^{19}$ may be considered instead as 'chronic low-to-moderate level of exposure to irritants' which may also induce asthma 3,6,7,26. Improvement of occupational exposure assessment during the entire working life is crucial $^{18}$ for a better understanding of the underlying mechanisms of irritant-induced asthma.

Therefore, for the new OAsJEM we excluded the 'Low risk' category and added specific irritants potentially at risk for asthma such as indoor cleaning agents, pesticides, endotoxin, aliphatic amines, acrylates, epoxy resins, persulphates/henna and organic solvents ${ }^{1,3}$. In the original $\mathrm{JEM}^{19}$, the literature at the time suggested that only industrial cleaning products, which contain highly reactive chemicals, mostly used in hospitals, were associated with asthma compared to cleaning products used at home or in offices. However, this has been refuted in recent literature ${ }^{2,3,12,13}$. Cleaning products and disinfectants are ubiquitous agents used in many places (such as hospital, office, home) and are now considered amongst the most common lung irritants ${ }^{3,10}$. Bleach is considered one of the main airway irritants among cleaning products and has been shown to induce acute irritative asthma earlier known as $\operatorname{RADS}^{17}$. Therefore, we have added Bleach as a sub-category of indoor cleaning products.

In addition, we excluded unspecific exposure categories: one a priori, agriculture, and five $a$ posteriori, bacteria, high peaks exposure to irritants, environmental tobacco smoke, highly reactive chemicals and dusts, gases, fumes in general. Agriculture workers are exposed to many agents associated with asthma ${ }^{3}$. These agents are often heterogeneous in term of mechanisms, as they may include both allergens, such as animal proteins, and irritants, such as pesticides and cleaning products. Large groups make it difficult to disentangle immunological or non-immunological mechanisms involved in occupational asthma. Following discussion within the expert group, it was decided $a$ posteriori to exclude Bacteria assessment because it was closely linked to endotoxins. In addition, we assumed that associations observed between bacteria and asthma may be explained by the presence of endotoxin. For high peak exposure to irritants many workers may be exposed such as freight handlers (gassed containers), police officers, farmers, animal care takers and animal transporters. However, only one study in which the Asthma-specific JEM was applied (a case-control study in Taiwan ${ }^{32}$, 
Table E1), found an association between irritant peaks and non-atopic asthma (odds ratio 4.2 [1.511.8]). Given that these peaks are induced during incidents, we considered that the JEM methodology is not the best approach to evaluate such exposure. In the same way, we decided to exclude the category Environmental Tobacco Smoke (ETS) from this new OAsJEM, because ETS assessment at job level might be of poor quality. Indeed, nowadays workers are unexposed to ETS in most workplaces, due to new smoking regulations, which may depend strongly on country and period. In addition, the large group highly reactive chemicals was evaluated through exposure assessment to eight specific agents (see Table 1). We have not evaluated exposure to dusts, gases and fumes as a group as it may not be relevant for asthma ${ }^{33}$, and it is especially important to define groups that allow disentangling sensitizers from irritating agents when studying underlying mechanism.

The study experts decided by consensus a posteriori (at step 2) to split 'mites' into 3 sub-categories and to add an evaluation of exposures to three sub-categories of pesticides (herbicides, insecticides, fungicides) based on the existing ALOHA JEM ${ }^{29,30}$.

\section{Evaluations of specific exposures}

Initial agreement between exposure evaluations of two experts (out of three) varied according to agents and was mostly fair to moderate, which is consistent with previous observations ${ }^{34,35}$. However, it is difficult to compare agreement for each specific agent to earlier findings as few comparable studies are available. The lack of initial congruence between experts underlines the importance of evaluating occupational exposures within a review team of at least two experts. For all the agents, a small percentage of jobs, out of 506, were classified exposed at high level. For half of the agents, less than $3 \%$ of the jobs were classified exposed at high or medium level. Compared to the original JEM, more jobs were classified exposed in general but less at high level with the OAsJEM. In the original JEM less than $3 \%$ of the jobs were classified exposed, except for three agents (textile, metals, exhaust fumes). As expected, the exposure assessment evaluated through the new OAsJEM was more specific for high level of exposure but more sensitive for medium level.

\section{Occupational Asthma-specific JEM}

Due to the lack of a gold standard, a formal validation of the OAsJEM estimates is not possible, which is a classical limitation of JEMs ${ }^{35,36}$. It has been suggested that updating a JEM may increase the validity $^{37}$. In addition, the development of the OAsJEM was based on recent literature and knowledge from multidisciplinary international experts, using a standardized procedure to take final decision by consensus. The OAsJEM has been updated from the original Asthma-specific JEM, which has been used in at least 51 publications to date, has been regarded as a valuable and robust standardised tool for evaluating exposure to asthmagens ${ }^{6,38}$. Published papers using this method allowed evaluating (1) efficiency and accuracy of the method but also (2) healthy worker effect, asthma- and allergy- related 
phenotypes, occupational exposures in other diseases such as lymphoma and autism, maternal occupational exposure and child health (Table E1, online supplement). The original Asthma-specific $\mathrm{JEM}^{19}$ was the sole JEM set-up to evaluated exposure to asthmagens in epidemiological surveys, and was previously adapted twice to take into account country-occupation specificities ${ }^{15,23}$.

This updated OAsJEM may improve the evaluation of associations between occupational exposures and asthma phenotypes in epidemiological studies and contribute to assessment of the burden of disease due to occupational asthmagens. In order to use the OAsJEM in optimal conditions, we recommend the following steps previously advised in the original $\mathrm{JEM}^{19}$ : first coding jobs by an experienced occupational coder and then applying the JEM with an expert re-evaluation step, blinded to disease status. In order to limit misclassification errors and to perform both job coding and the expert re-evaluation step in an optimal way, recording well described tasks for each job is crucial in epidemiological surveys. During the expert re-evaluation step it is important to keep in mind that the OAsJEM was built to promote specificity for high level of exposure to asthmagen. Other important points include (i) taking into account, for some identified exposures, time of exposure occurrence and in which country, (ii) following the recommendations of the re-evaluation step written for each job, for both ISCO code and exposure assessment checking and (iii) performing the expert re-evaluation step with at least two experts, independently. In the update, we have tried to limit the number of expert reevaluations to improve feasibility of this additional step. Some analyses ${ }^{7,33}$ performed in large cohorts suggest that it is possible to use the JEM without an expert re-evaluation step, especially after exclusion of poorly defined or heterogeneous jobs (such as those classified as needing an expert reevaluation step). In addition, information regarding task descriptions, specific workplace conditions (ventilation, personal respiratory protection use ... ) or target tasks, as recorded in occupational epidemiology studies $^{39}$, may improve evaluations of exposure assessment from the OAsJEM agents by decreasing misclassification errors at the re-evaluation step.

The new OAsJEM is available for free on the website (http://asthmajem.vjf.inserm.fr/). In order to keep it up to date, we would appreciate receiving a notification through the website or by email to the corresponding author, when the application of the OAsJEM in an epidemiological study will result in a publication.

\section{Strengths and limitations}

The major strength of this OAsJEM is that initial exposure assessments were undertaken by experts independently from each other and final evaluations were taken by consensus, through a standardized procedure. Furthermore, we incorporated improvements based on other JEMs, such as ALOHA and Northern-JEM developed by experts from our team, to evaluate exposure assessment to pesticides and acrylates or epoxy resins, respectively. We finally checked that for all jobs with an expert reevaluation step the default exposure assessment for a specific agent was the most likely one. In 
addition, the standardisation of an expert re-evaluation step with precisely written recommendations may be helpful for non-experts. Furthermore, this method has previously shown its efficiency and usefulness to evaluate associations between occupational exposures and diseases (online supplement Table E1).

The present method also has limitations. As with any JEM, non-differential misclassification remains but is somewhat reduced by incorporating up-to-date knowledge from eight experts for the evaluated agents. We acknowledge that the additional expert assessment step may be time and money consuming which may induce difficulties to apply the method in large epidemiological studies. However, the expert step is mostly intended to improve precision of exposure estimates in studies of moderate size and limited power. Our list of 30 asthmagens was not exhaustive but in accordance with most agents identified in recent papers ${ }^{1,5,40}$. This method does not intend to identify new occupational risks, but may improve estimates for the most common agents known or suspected to have an adverse effect on work-related asthma till now ${ }^{3,4}$. We developed the OAsJEM for the 2- to 4-digit ISCO-88 job codes, the most commonly used classification in Europe ${ }^{34}$. However, ISCO-88 classification was originally defined for economic statistical purposes, which may not be optimal for evaluation of occupational exposures. For example, the 5-digit ISCO-68 is a much more detailed classification and might be more accurate to evaluate occupational exposures, in spite of its lack of representation of more contemporaneous jobs. In future developments, it would be useful to adapt the OAsJEM for ISCO-68 and potentially ISCO-08 job codes. For certain agents, OAsJEM exposure assessment may be improved by applying agent-specific JEMs with quantitative exposure assessment, for example for endotoxin $^{41}$, wood dusts $^{42}$, solvents and exhaust fumes (FINJEM). Applying the new OAsJEM in ongoing prospective epidemiological respiratory health studies, such as EGEA ${ }^{43}$ and ECRHS ${ }^{38}$ studies, in which the original JEM has been applied will allow for comparisons of the original and updated Asthma JEMs.

\section{Conclusions}

In conclusion, we have developed an updated OAsJEM that allows evaluation of retrospective occupational exposure assessment to 30 specific asthmagens and irritants for all ISCO-88 job codes. This OAsJEM, developed to improve occupational asthmagen exposure assessment, may improve the evaluation of the association between occupational exposures and asthma phenotypes in epidemiological studies and contribute to assessment of the burden of work-related asthma. 
Acknowledgements: We thank Catherine Quinot (Inserm U1168) for checking analysis.

Contributors: NLM contributed to the study design, acquisition, analysis and interpretation of the data, drafting and revising the manuscript. JPZ and HK contributed to the study design, analysis and interpretation of the data and critically revised the manuscript. OD, TL, EA, LL, VS, GB contributed to analysis and interpretation of the data and critically revised the manuscript. All authors approved the final version of the manuscript.

Fundings: PHC Van Gogh programme, EP-Nuffic, Campus France 33653RF (2015-2016); 7th programme-EU (Marie-Curie) [FP7/2007-2013], REA (PCOFUND-GA-2013-609102), PRESTIGE2015-3-0029 - Campus France. ISGlobal is a member of the CERCA Programme, Generalitat de Catalunya.

Competing interests: N Le Moual reports grants from PHC Van Gogh programme, Campus France ; O Dumas reports grants from Prestige, Campus France; H Kromhout reports grants from PHC Van Gogh programme, EP-Nuffic, during the conduct of the study ; None declared for others co-authors. 


\section{REFERENCES}

1. Tarlo SM, Lemiere C. Occupational asthma. N. Engl. J. Med. 2014;370(7):640-649.

2. Siracusa A, De Blay F, Folletti l, et al. Asthma and exposure to cleaning products - a European Academy of Allergy and Clinical Immunology task force consensus statement. Allergy. 2013;68(12):1532-1545.

3. Dumas O, Le Moual N. Do chronic workplace irritant exposures cause asthma? Curr. Opin. Allergy Clin. Immunol. 2016;16:75-85.

4. Cartier A. New causes of immunologic occupational asthma, 2012-2014. Curr. Opin. Allergy Clin. Immunol. 2015;15(2):117-123.

5. Vandenplas O, Wiszniewska M, Raulf M, et al. EAACl position paper: irritant-induced asthma. Allergy. 2014;69(9):1141-1153.

6. Ghosh RE, Cullinan P, Fishwick D, et al. Asthma and occupation in the 1958 birth cohort. Thorax. 2013;68(4):365-371.

7. Dumas $\mathrm{O}$, Laurent $\mathrm{E}$, Bousquet J, et al. Occupational irritants and asthma: an Estonian crosssectional study of 34,000 adults. Eur Respir J. 2014;44:647-656.

8. Cullinan $P$, Munoz $X$, Suojalehto $H$, et al. Occupational lung diseases: from old and novel exposures to effective preventive strategies. Lancet Respir Med. 2017;5(5):445-455.

9. Andersson E, Knutsson A, Hagberg $S$, et al. Incidence of asthma among workers exposed to sulphur dioxide and other irritant gases. Eur. Respir. J. 2006;27(4):720-725.

10. Malo JL, Tarlo SM, Sastre J, et al. An official American Thoracic Society Workshop Report: presentations and discussion of the fifth Jack Pepys Workshop on Asthma in the Workplace. Comparisons between asthma in the workplace and non-work-related asthma. Ann Am Thorac Soc. 2015;12(7):S99-S110.

11. Chan-Yeung M, Malo JL, Tarlo SM, et al. Proceedings of the first Jack Pepys occupational asthma symposium. Am. J. Respir. Crit. Care Med. 2003;167(3):450-471.

12. Folletti I, Siracusa A, Paolocci G. Update on asthma and cleaning agents. Curr. Opin. Allergy Clin. Immunol. 2017;17(2):90-95.

13. De Matteis S, Cullinan P. Occupational asthma in cleaners: a challenging black box. Occup. Environ. Med. 2015;72(11):755-756.

14. Mamane A, Baldi I, Tessier JF, Raherison C, Bouvier G. Occupational exposure to pesticides and respiratory health. Eur Respir Rev. 2015;24(136):306-319.

15. Lillienberg L, Andersson E, Janson C, et al. Occupational exposure and new-onset asthma in a population-based study in Northern Europe (RHINE). Ann. Occup. Hyg. 2013;57(4):482-492.

16. LeVan TD, Koh WP, Lee HP, Koh D, Yu MC, London SJ. Vapor, dust, and smoke exposure in relation to adult-onset asthma and chronic respiratory symptoms: the Singapore Chinese Health Study. Am.J.Epidemiol. 2006;163(12):1118-1128.

17. Lemiere $C$, Ameille J, Boschetto $P$, Labrecque $M$, Pralong JA. Occupational asthma: new deleterious agents at the workplace. Clin. Chest Med. 2012;33(3):519-530.

18. Heederik D. Cleaning agents and disinfectants: moving from recognition to action and prevention. Clin. Exp. Allergy. 2014;44(4):472-474.

19. Kennedy SM, Le Moual N, Choudat D, Kauffmann F. Development of an asthma specific job exposure matrix and its application in the epidemiological study of genetics and environment in asthma (EGEA). Occup Environ Med. 2000;57:635-641, available from: http://asthmajem.vjf.inserm.fr/. Date last accessed: 20 October, 2017.

20. Chan-Yeung M, Malo JL. Table of major inducers of occupational asthma. In: Bernstein IL, Chan-Yeung M, Malo JL, Bernstein DI, editors., eds. Asthma in the workplace. New-York: Marcel Dekker, Inc; 1993:595-623.

21. Zock JP, Cavalle N, Kromhout $\mathrm{H}$, et al. Evaluation of specific occupational asthma risks in a community-based study with special reference to single and multiple exposures. J. Expo. Anal. Environ. Epidemiol. 2004;14(5):397-403. 
22. Abrahamsen R, Fell AK, Svendsen MV, et al. Association of respiratory symptoms and asthma with occupational exposures: findings from a population-based cross-sectional survey in Telemark, Norway. BMJ Open. 2017;7(3):e014018.

23. Tagiyeva N, Devereux G, Semple S, et al. Parental occupation is a risk factor for childhood wheeze and asthma. Eur. Respir. J. 2010;35(5):987-993.

24. Tagiyeva N, Teo E, Fielding S, Devereux G, Semple S, Douglas G. Occupational exposure to asthmagens and adult onset wheeze and lung function in people who did not have childhood wheeze: A 50-year cohort study. Environ Int. 2016;94:60-68.

25. International standard classification of occupations. Geneva, Switzerland1988.

26. Lillienberg L, Dahlman-Hoglund A, Schioler L, Toren K, Andersson E. Exposures and asthma outcomes using two different job exposure matrices in a general population study in northern Europe. Ann. Occup. Hyg. 2014;58(4):469-481.

27. Schlunssen V, Sigsgaard T, Raulf-Heimsoth M, Kespohl S. Workplace exposure to wood dust and the prevalence of wood-specific sensitization. Allergologie, Jahrgang. 2012;35:402-412.

28. Quirce S, Bernstein JA. Old and new causes of occupational asthma. Immunol. Allergy Clin. North Am. 2011;31(4):677-698.

29. Matheson MC, Benke G, Raven J, et al. Biological dust exposure in the workplace is a risk factor for chronic obstructive pulmonary disease. Thorax. 2005;60(8):645-651.

30. Sunyer J, Kogevinas $M$, Kromhout $H$, et al. Pulmonary ventilatory defects and occupational exposures in a population-based study in Spain. Spanish group of the european community respiratory health survey. Am.J.Respir.Crit Care Med. 1998;157(2):512-517.

31. Landis JR, Koch GG. The measurement of observer agreement for categorical data. Biometrics. 1977;33(1):159-174.

32. Wang TN, Lin MC, Wu CC, et al. Risks of exposure to occupational asthmogens in atopic and nonatopic asthma: a case-control study in Taiwan. Am. J. Respir. Crit. Care Med. 2010;182(11):1369-1376.

33. Le Moual N, Kennedy SM, Kauffmann F. Occupational exposures and asthma in 14,000 adults from the general population. Am J Epidemiol. 2004;160(11):1108-1116.

34. Martin-Bustamante $M$, Oliete-Canela A, Dieguez-Rodriguez $M$, et al. Job-exposure matrix for the assessment of alkylphenolic compounds. Occup. Environ. Med. 2017;74(1):52-58.

35. Teschke $\mathrm{K}$, Olshan AF, Daniels $\mathrm{JL}$, et al. Occupational exposure assessment in case-control studies: opportunities for improvement. Occup Environ Med. 2002;59(9):575-593.

36. McGuire V, Nelson LM, Koepsell TD, Checkoway H, Longstreth WT, Jr. Assessment of occupational exposures in community-based case-control studies. Annu. Rev. Public Health. 1998;19:35-53.

37. Kauppinen $\mathrm{T}$, Heikkila $\mathrm{P}$, Plato $\mathrm{N}$, et al. Construction of job-exposure matrices for the Nordic Occupational Cancer Study (NOCCA). Acta Oncol. 2009;48(5):791-800.

38. Kogevinas $M$, Zock JP, Jarvis $D$, et al. Exposure to substances in the workplace and new-onset asthma: an international prospective population-based study (ECRHS-II). Lancet. 2007;370(9584):336-341.

39. Clavel J, Glass DC, Cordier S, Hemon D. Standardization in the retrospective evaluation by experts of occupational exposure to organic solvents in a population-based case-control study. Int.J.Epidemiol. 1993;22 Suppl 2:S121-S126.

40. Crewe J, Carey R, Glass D, et al. A comprehensive list of asthmagens to inform health interventions in the Australian workplace. Aust. N. Z. J. Public Health. 2016;40(2):170-173.

41. Basinas I, Wouters IM, Sigsgaard T, et al. O46-4 Development of a quantitative job exposure matrix for endotoxin exposure in agriculture. Occup.Environ.Med. 2016;73:A88.

42. Basinas I, Liukkonen T, Sigsgaard T, et al. P096 Statistical modelling and development of a quantitative job exposure matrix for wood dust in the wood manufacturing industry. Occup Environ Med. 2016;73:A152-A153. 
43. Le Moual N, Siroux V, Pin I, Kauffmann F, Kennedy SM, on behalf of the Epidemiological Study on the Genetics and Environment of Asthma. Asthma severity and exposure to occupational asthmogens. Am J Respir Crit Care Med. 2005;172(4):440-445. 